14

\title{
Бифуркационный анализ регуляции активности ноцицептивных нейронов
}

\author{
(C) O.E. Дик
}

Научно-исследовательский институт фризиологии им. И.П. Павлова РАН, 199034 Санкт-Петербург, Россия

e-mail: dickviola@gmail.com

(Поступило в Редакцию 3 апреля 2017 г.)

На модели мембраны ноцицептивного нейрона дорсальных ганглиев крысы рассмотрена задача анализа регуляции ноцицептивных сигналов под действием 5-гидрокси- $\gamma$-пирон-2-карбоновой кислоты, являющейся лекарственной субстанцией анальгетика аноцептина. Использован метод бифуркационного анализа, позволяющий установить соотношения между значениями параметров модели и типом решения задачи до и после изменения параметров, связанных с анальгетической модуляцией.

DOI: 10.21883/JTF.2017.11.45140.2277

\section{Введение}

Болевая чувствительность активируется периферическими ноцицепторами, передающими сигналы к ноцицептивным нейронам дорсальных ганглиев [1]. Нормально низкий уровень электрической активности в нейронах дорсальных ганглиев, отмечаемый, как правило, в 75\% нейронов в виде медленных нерегулярных одиночных разрядов, многократно усиливается, когда эти разряды трансформируются в пачечную активность с высокой частотой импульсов внутри пачки [2]. Это резко усиливает вероятность возникновения эктопического пачечного разряда, связанного с передачей болевого сигнала [3]. Пачечная активность (при которой пачки импульсов рассматриваются как единица нейрональной информации) значительно повышает надежность передачи сигнала между нейронами [4]. Поэтому неудивительно, что пачечные разряды могут играть важную роль в инициации восприятия болевого повреждающего воздействия.

Считается, что для нейронов дорсальных ганглиев характерен потенциало-зависимый механизм возникновения пачечной активности [3,5]. Однако однозначный ответ на вопрос о том, какие именно ионные каналы входящих токов вносят основной вклад в возникновение пачечных разрядов при повреждающем болевом воздействии, до сих пор не получен. Известно, что в нейронах дорсальных ганглиев большого диаметра взрослого млекопитающего быстрые $\mathrm{Na}^{+}$-токи генерируются прохождением ионов $\mathrm{Na}^{+}$через $\mathrm{Na}_{V} 1.1, \mathrm{Na}_{V} 1.3$, $\mathrm{Na}_{V} 1.7$ тетродотоксин-чувствительные каналы [6,7]. Кроме этого, эти каналы могут генерировать задержанный $\mathrm{Na}^{+}$-ток с постоянными времени воротных процессов порядка 5-10 ms [8,9]. Задержанные натриевые токи также поддерживаются прохождением ионов $\mathrm{Na}^{+}$через тетродотоксин-устойчивые $\mathrm{Na}_{V} 1.8$-каналы [10] и $\mathrm{Na}_{V} 1.5$ каналы [11]. Перерезка аксонов значительно усиливает электрогенез в нейронах дорсальных ганглиев [12]. Однако множество медиаторов, присутствующих в поврежденных нервах, способно различным образом изменять кинетику входящих токов, и неизвестно, отражается ли повреждение в сдвиге относительных популяций типов специфических натриевых каналов или только в изменении кинетики отдельных каналов [13].

Ключевая роль в генерации болевой чувствительности отводится медленным натриевым каналам $\mathrm{Na}_{V} 1.8$ [14], поскольку повышение синтеза этих каналов связано с гипервозбудимостью ноцицептивных нейронов и возникновением нейропатической боли [15]. Модуляция активности этих каналов медиаторами воспаления может приводить к патологическому состоянию, связанному с повышением болевой чувствительности [16]. В работах [17] показано, что такое состояние может быть устранено действием химических реагентов, оказывающих влияние на $\mathrm{Na}_{V} 1.8$-каналы.

Цель работы - получение ответа на вопрос о том, какие именно параметры натриевых каналов $\mathrm{Na}_{V} 1.8$ ответственны за выключение эктопических пачечных разрядов и, следовательно, оказывают наибольшее влияние на передачу болевого сигнала. Для этого в настоящей работе используется модельный подход для установления связей между значениями параметров, описывающих импульсную активность в ноцицептивных нейронах, и типом решения задачи до и после изменения параметров, связанных с анальгетической модуляцией.

\section{Модель}

Модель мембраны ноцицептивного нейрона может быть описана системой уравнений [18]

$$
\begin{aligned}
\frac{d E}{d t}= & \left(I-I_{N a f}(m, h, E)-I_{N a I}(b, E)-I_{\mathrm{K}}(n, E)\right. \\
& \left.-I_{L}(E)-I_{N a s}(s, r, E)\right) / c_{m}, \\
\frac{d x}{d t}= & \left(x_{\infty}(E)-x\right) / \tau_{x}(E), \quad x=m, h, n, b, s, r,
\end{aligned}
$$

где $E$ - величина мембранного потенциала, $I$ - величина стимулирующего тока, $I_{\mathrm{K}}$ - задержанный калиевый 
ток, $I_{L}$ - ток утечки, $I_{N a f}-$ быстро активирующийся и инактивирующийся тетродотоксин-чувствительный натриевый ток, $I_{N a I}$ - промежуточный, быстро активирующийся и медленнее инактивирующийся натриевый ток, $I_{N a s}$ - еще медленнее инактивирующийся тетродотоксин-устойчивый натриевый ток, генерируемые $\mathrm{Na}_{V} 1.1-, \mathrm{Na}_{V} 1.7-$ и $N a_{V} 1.8$-каналами соответственно

$$
\begin{gathered}
I_{N a f}=g_{\mathrm{Na}} m^{3} h\left(E-E_{\mathrm{Na}}\right), \\
I_{N a I}(b, E)=g_{N a I} m_{I \infty}(E) b\left(E-E_{\mathrm{Na}}\right), \\
I_{\mathrm{K}}(n, E)=g_{\mathrm{K}} n\left(E-E_{\mathrm{K}}\right), \\
I_{L}(E)=g_{L}\left(E-E_{L}\right), \quad I_{N a s}(s, r, E)=g_{N a s} s^{3} r\left(E-E_{\mathrm{Na}}\right),
\end{gathered}
$$

где переменные $m, h, n, b, s, r$ описывают воротные характеристики процессов активации и инактивации ионных каналов, константы $c_{m}=1 \mu \mathrm{F} / \mathrm{cm}^{2} ; \quad g_{N a I}=$ $=27 \mathrm{mS} / \mathrm{cm}^{2}, g_{\mathrm{K}}=1.5 \mathrm{mS} / \mathrm{cm}^{2}, g_{L}=1.4 \mathrm{mS} / \mathrm{cm}^{2}, g_{\text {Nas }}=$ $=5 \mathrm{mS} / \mathrm{cm}^{2}$ определяют емкость мембраны, максимальные проводимости каналов, $E_{\mathrm{Na}}=62 \mathrm{mV}, E_{\mathrm{K}}=-94 \mathrm{mV}$, $E_{L}=-77 \mathrm{mV}$ - равновесные потенциалы для $\mathrm{Na}^{+}-, \mathrm{K}^{+}$ионов и ионов утечки. Потенциал-зависимые стационарные и динамические характеристики воротных процессов всех каналов, кроме каналов $\mathrm{Na}_{V} 1.8$, определяются функциями

$$
\begin{gathered}
m_{\infty}=1 /(1+\exp (-(34.1+E) / 9.1)) \\
h_{\infty}=1 /(1+\exp (56.4+E) / 7.2) \\
m_{I \infty}=1 /(1+\exp (-(25.3+E) / 9.1)) \\
b_{\infty}=1 /(1+\exp (72.5+E) / 8) \\
n_{\infty}=1 /(1+\exp (-(9.2+E) / 16)) \\
\tau_{m}=0.01+0.11 \exp \left(-0.5((E+28.7) / 25.5)^{2}\right) \\
\tau_{h}=0.24+1.63 \exp \left(-0.5((E+61.9) / 15.3)^{2}\right) \\
\tau_{m_{I}}=0, \quad \tau_{b}=0.22 \exp (-0.07 E) \\
\tau_{n}=-23+69.4 \exp (-0.01 E)
\end{gathered}
$$

Функции $s_{\infty}(E), r_{\infty}(E), \tau_{s}(E)$ и $\tau_{r}(E)$ для активации и инактивации медленных тетродотоксин-устойчивых натриевых каналов $\mathrm{Na}_{V} 1.8$ были определены ранее по экспериментально зарегистрированным медленным натриевым токам [19]:

$$
\begin{gathered}
\tau_{s}(E)=1 /\left(\exp \left(a_{1} E+b_{1}\right)+\exp \left(a_{2} E+b_{2}\right)\right), \\
s_{\infty}(E)=\exp \left(a_{1} E+b_{1}\right) /\left(\exp \left(a_{1} E+b_{1}\right)+\exp \left(a_{2} E+b_{2}\right)\right), \\
\tau_{r}(E)=1 /\left(\exp \left(a_{3} E+b_{3}\right)+\exp \left(a_{4} E+b_{4}\right)\right), \\
r_{\infty}(E)=\exp \left(a_{3} E+b_{3}\right) /\left(\exp \left(a_{3} E+b_{3}\right)+\exp \left(a_{4} E+b_{3}\right)\right),
\end{gathered}
$$

где

$$
\begin{gathered}
a_{1}=0.043 \pm 0.002, \quad b_{1}=-2.22 \pm 0.11 \\
a_{2}=-0.048 \pm 0.003, \quad b_{2}=-4.33 \pm 0.23
\end{gathered}
$$

$$
\begin{gathered}
a_{3}=-0.032 \pm 0.002, \quad b_{3}=-6.41 \pm 0.41, \\
a_{4}=0.056 \pm 0.005, \quad b_{4}=-5.62 \pm 0.31 .
\end{gathered}
$$

На основании полученных зависимостей величина эффективного заряда $Z_{\mathrm{eff}}$, связанного с конформационным изменением активационной воротной системы при переносе иона через мембрану (в единицах заряда электрона), может быть определена как $Z_{\mathrm{eff}}=(3 k T / e)\left(a_{1}-a_{2}\right)$, где $\bar{e}-$ абсолютная величина заряда электрона, $k-$ постоянная Больцмана, $T$ - абсолютная температура, коэффициент 3 учитывает трехбарьерность модели $\left(s^{3}\right)$ с одним открытым и тремя закрытыми состояниями. Рассчитанная величина эффективного заряда составляет $Z_{\text {eff }}=6.9 \pm 0.3$ [19]. Изменения в зависимостях активационной воротной структуры после воздействия на мембрану нейрона 5-гидрокси- $\gamma$-пирон-2-карбоновой (коменовой) кислоты, являющейся лекарственной субстанцией анальгетика аноцептина [20] в концентрации $100 \mathrm{nmol} / \mathrm{l}$, соответствуют уменьшению эффективного заряда до величины $Z_{\mathrm{eff}}=4.7 \pm 0.2$ и изменению характеристик активационной воротной системы до значений

$$
\begin{aligned}
& a_{1}=0.047 \pm 0.003, \quad b_{1}=-2.71 \pm 0.16 \\
& a_{2}=-0.015 \pm 0.002, \quad b_{2}=-4.05 \pm 0.27[19]
\end{aligned}
$$

\section{Разделение пространства параметров модели на области качественно различных решений}

Для установления связей между значениями параметров модели, описывающей импульсную активность, и типом решения исследуемой модели до и после ее анальгетической модуляции необходимо найти границу, разделяющую множество значений параметров на области качественно различных типов устойчивых решений, при которых наблюдается периодическая импульсация и при которых колебания отсутствуют или являются затухающими. Для построения этой границы применялись метод бифуркационного анализа [21] и пакет программ MATCONT [22]. Численные решения системы (1) внутри полученной области устойчивых периодических решений находились методом Рунге-Кутты четвертого порядка, модифицированным алгоритмом Гира с выбором переменного шага интегрирования.

Так как в режиме пачечной активности переходы между активной фазой, в которой наблюдаются пачечные разряды, и фазой их отсутствия связаны с взаимодействием быстрых и медленных колебаний мембранного потенциала, для нахождения границы области пачечной активности было применено разложение исходной системы на медленную и быструю. В рамках такого подхода возникновение быстрой компоненты пачечного разряда рассматривается как результат бифуркации быстрой подсистемы при изменении значений медленных переменных, которые выполняют роль бифуркационных параметров [23]. В связи с тем, что калиевый и 


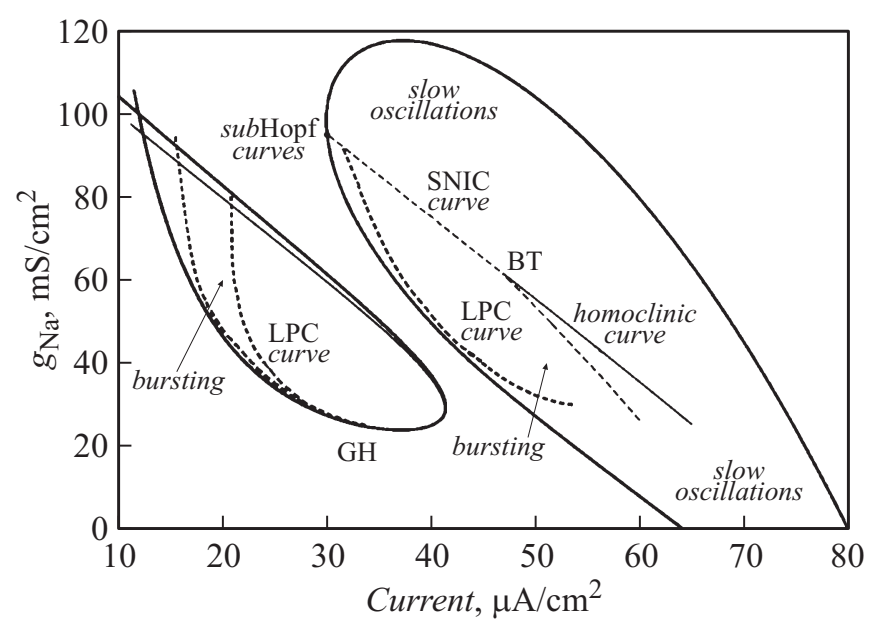

Рис. 1. Двухпараметрические бифуркационные диаграммы полной системы в плоскости $\left(g_{\mathrm{Na}}, I\right)$.

медленный натриевый токи имеют постоянные времени $\left(\tau_{n}\right.$ и $\left.\tau_{r}\right)$, значительно превышающие эти величины для других токов, в качестве быстрой подсистемы будем рассматривать систему

$$
\begin{aligned}
\frac{d E}{d t}= & \left(I-I_{N a f}(m, h, E)-I_{N a I}(b, E)-I_{\mathrm{K}}(n, E)\right. \\
& \left.-I_{L}(E)-I_{N a s}(s, r, E)\right) / c_{m}, \\
\frac{d x}{d t}= & \left(x_{\infty}(E)-x\right) / \tau_{x}(E), \quad x=m, h, b, s,
\end{aligned}
$$

в которой $n$ и $r$ являются медленно меняющимися бифуркационными параметрами.

Медленную систему запишем в виде

$$
\begin{gathered}
I-I_{N a f}(m, h, E)-I_{N a I}(b, E)-I_{\mathrm{K}}(n, E) \\
\quad-I_{L}(E)-I_{N a s}(s, r, E)=0, \\
\frac{d x}{d t}=\left(x_{\infty}(E)-x\right) / \tau_{x}(E), \quad x=n, r .
\end{gathered}
$$

Начало активной фазы пачечного разряда соответствует потере устойчивости стационарного состояния быстрой подсистемы, а окончание активной фазы соответствует потере устойчивости предельного цикла быстрой подсистемы [24].

\section{Результаты}

На рис. 1 на плоскости $\left(I, g_{\mathrm{Na}}\right)$ представлены двухпараметрические бифуркационные диаграммы полной системы до модификации натриевых $\mathrm{Na}_{V} 1.8$-каналов. Сплошные жирные линии являются кривыми бифуркации Андронова-Хопфа и определяют границы, разделяющие плоскость параметров $\left(I, g_{\mathrm{Na}}\right)$ на области устойчивых и неустойчивых стационарных состояний полной системы, и ограничивают области повторных ответов.
Большая часть границы левой области представлена кривой субкритической бифуркации Хопфа, в точках которой происходит жесткое возбуждение и стационарное состояние теряет устойчивость, что приводит к возникновению предельного цикла большой амплитуды. Левая граница правой области также соответствует субкритической бифуркации Хопфа, а правая граница соответствует суперкритической бифуркации Хопфа, в точках которой наблюдается мягкое возбуждение, стационарное состояние плавно теряет устойчивость, и возникает устойчивый предельный цикл малой амплитуды.

Область пачечной активности внутри левой области стационарных состояний полной системы ограничена кривой бифуркации Андронова-Хопфа быстрой подсистемы (сплошная линия внутри кривой бифуркации Андронова-Хопфа полной системы) и кривыми седлоузловых бифуркаций предельного цикла (LPC), отмеченными на рис. 1 штрихпунктирными линиями и начинающимися в точке обобщенной буфуркации Хопфа (GH). Область пачечной активности внутри правой области стационарных состояний полной системы ограничена кривой седло-узловой бифуркации предельного цикла (LPC), отмеченной толстой штрихпунктирной линией, и кривой бифуркации седло-узел на инвариантном круге (SNIC кривой, обозначенной тонкой штрихпунктирной линией).

Отметим, что при значениях параметров модели, соответствующих контрольным условиям $\left(Z_{\mathrm{eff}}=\right.$ $=6.9 \pm 0.3)$, существуют две области ритмической активности, и разряды наблюдаются при значениях тока $10<I<80\left(\mu \mathrm{A} / \mathrm{cm}^{2}\right)$. Наличие двух областей ритмической активности объясняется наличием четырех вместо двух точек бифуркации Андронова-Хопфа для каждого значения параметра $g_{\mathrm{Na}}$.

Примеры колебаний полной системы до модификации натриевых каналов $\mathrm{Na}_{V} 1.8$ даны на рис. 2. При значениях параметров $g_{\mathrm{Na}}=39.71 \mathrm{mS} / \mathrm{cm}^{2}$ и $I=23.9 \mu \mathrm{A} / \mathrm{cm}^{2}$ наблюдается пачечная активность в форме дуплетов с малой амплитудой подпороговых колебаний между пачками (рис. $2, a)$. При значениях $g_{\mathrm{Na}}=39.71 \mathrm{mS} / \mathrm{cm}^{2}$ и $I=24.5 \mu \mathrm{A} / \mathrm{cm}^{2}$ пачечная активность становится нерегулярной в результате изменения интервала между спайками (рис. $2, b)$. При $g_{\mathrm{Na}}=73 \mathrm{mS} / \mathrm{cm}^{2}$ и $I=41 \mu \mathrm{A} / \mathrm{cm}^{2}$ во время пачечной активности амплитуда спайков в пачке постепенно уменьшается, а частота растет (рис. 2,c). Особенностью этих колебаний является большая амплитуда в интервалах между пачками. При $g_{\mathrm{Na}}=60 \mathrm{mS} / \mathrm{cm}^{2}$ и $I=50.47 \mu \mathrm{A} / \mathrm{cm}^{2}$ пачечная активность исчезает, и остаются только медленные колебания (рис. 2,d). Таким образом, две построенные области характеризуются различной динамикой колебаний, при этом внутри каждой области существует только один тип пачечной активности (дуплеты в левой области и пачечные разряды с большим количеством разрядов внутри пачки в правой области).

Рассмотрим один из возможных механизмов возникновения пачечной активности в форме дуплетов в модели 

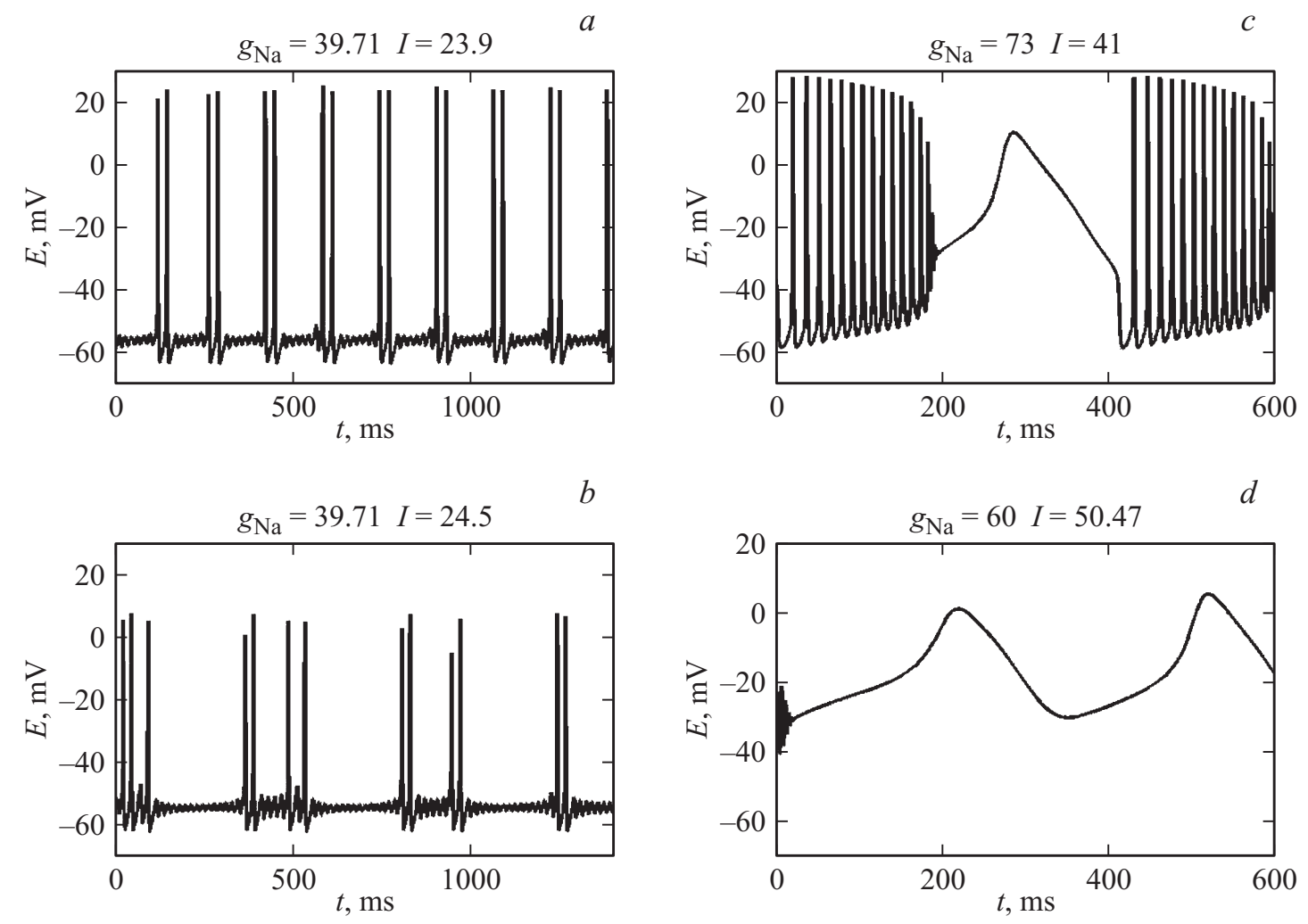

Рис. 2. Колебания полной системы для $g_{\text {Na }}$ и $I$, соответствующих левой $(a, b)$ и правой $(c, d)$ областям на рис. 1 .
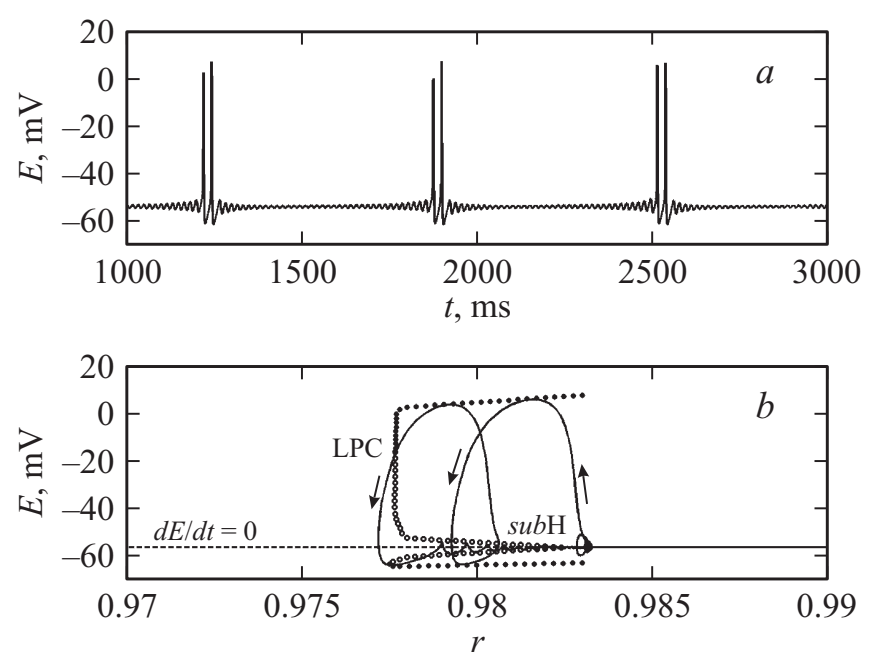

Рис. 3. Пример пачки типа Хопф/складка для значений параметров $g_{\mathrm{Na}}=39.71 \mathrm{mS} / \mathrm{cm}^{2}, I=22.4 \mu \mathrm{A} / \mathrm{cm}^{2}$. Траектория полной системы (сплошная кривая) изображена в проекции на плоскость $(r, E)$ вместе с бифуркационной диаграммой быстрой системы.

нейрона дорсального ганглия. На однопараметрической бифуркационной диаграмме, построенной при значениях параметров $g_{\mathrm{Na}}=39.71 \mathrm{mS} / \mathrm{cm}^{2}, I=22.4 \mu \mathrm{A} / \mathrm{cm}^{2}$ и представленной на рис. 3, величина $r$ является бифуркационным параметром. Бифуркационная диаграмма включает в себя ветви стационарных состояний и периодических орбит. Сплошные и штрихпунктирные кривые определяют устойчивые и неустойчивые стационарные состояния. Неустойчивые периодические орбиты обозначены незаштрихованными кружками, устойчивые орбиты - заштрихованными, отражающими максимальные и минимальные значения потенциала $E$ орбиты. В интервале между пачками траектория полной системы (сплошная кривая) движется в сторону уменьшения параметра $r$ вдоль ветви стационарных состояний быстрой подсистемы. Активная фаза пачечной активности начинается, когда траектория проходит через точку (subH) субкритической бифуркации Андронова-Хопфа быстрой подсистемы. В этой точке стационарное состояние полной системы теряет устойчивость, и система скачком переходит в режим колебаний с нарастающей амплитудой до тех пор, пока траектория системы не достигнет ветви устойчивых периодических колебаний большой амплитуды. Затем траектория движется влево, пока не достигнет седло-узловой бифуркации предельного цикла (обозначенной как LPC-точка — fold limit cycle bifurcation). Наконец, траектория полной системы возвращается к $E$-нульклине $(d E / d t=0)$ и затухающим колебаниям. Это так называемый тип пачки Хопф/складка (subHopf/fold cycle burster) по топологической классификации моделей пачечной активности Ижикевича [25], так как активная фаза пачечной активности начинается в точке субкритической бифуркации Андронова-Хопфа и заканчивается в седло-узловой бифуркации предельного 


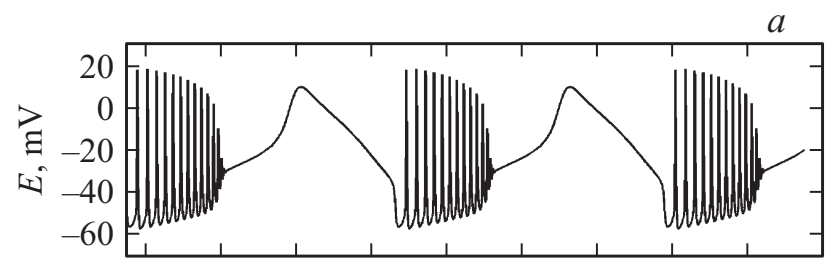

1100120013001400150016001700180019002000 $t, \mathrm{~ms}$

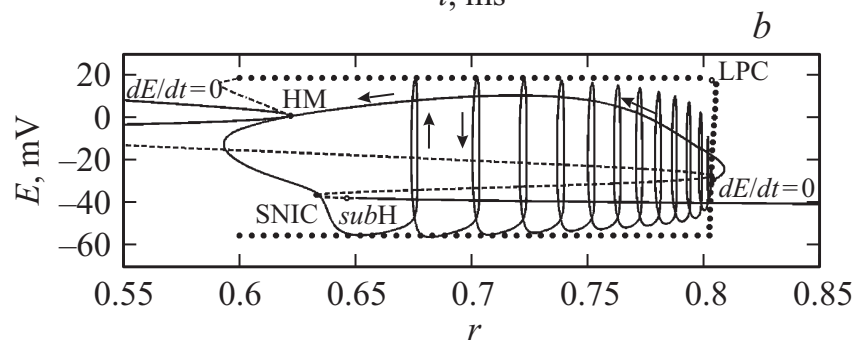

Рис. 4. Пример пачки типа круг/складка через гомоклиническую петлю гистерезиса для параметров $g_{\mathrm{Na}}=63.59 \mathrm{mS} / \mathrm{cm}^{2}$, $I=44.3 \mu \mathrm{A} / \mathrm{cm}^{2}$.
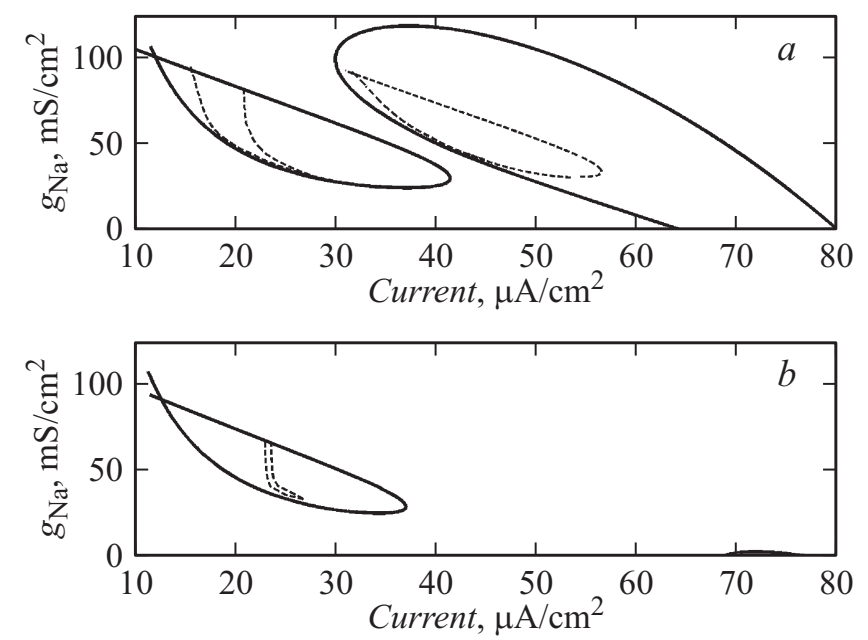

Рис. 5. Сравнение областей пачечной активности до (a) и после $(b)$ модификации натриевых $\mathrm{Na}_{V} 1.8$-каналов. Области ограничены штриховыми линиями.

цикла быстрой подсистемы. Именно такой тип пачечной активности развивается в поврежденных сенсорных нейронах после травмы и инициируется, вероятно, подпороговыми колебаниями мембранного потенциала вокруг деполяризованного состояния [26].

Другой возможный механизм возникновения пачечной активности в модели нейрона дорсальных ганглиев показан на рис. 4 для значений параметров $g_{\mathrm{Na}}=63.59 \mathrm{mS} / \mathrm{cm}^{2}, I=44.3 \mu \mathrm{A} / \mathrm{cm}^{2}$. Активная фаза пачечной активности в этом случае начинается, когда траектория полной системы (сплошная линия) проходит через точку бифуркации седло-узел на инвариантном круге (saddle-node bifurcation on invariant circle) быстрой подсистемы (точка, обозначенная SNIC, которая находится вблизи точки субкритической бифуркации Андронова-Хопфа (точка subH)). Такая бифуркация приводит к появлению устойчивого предельного цикла большой амплитуды. В результате в полной системе устанавливается режим колебаний с возрастающей частотой, и траектория полной системы сдвигается вправо до тех пор, пока не достигнет седло-узловой бифуркации предельного цикла (LPC-точки). После этого траектория полной системы касается $E$-нульклины $(d E / d t=0)$ в точке гомоклинической бифуркации седла (НМ), что приводит к рождению другого предельного цикла с очень большим периодом. Затем траектория полной системы снова достигает точки бифуркации седло-узел на инвариантном круге (SNIC), и пачечная активность возобновляется. Таким образом, это вариант пачки типа круг/складка через гомоклиническую петлю гистерезиса circle/fold cycle via homoclinic/circle hysteresis loop (cyclecycle burster) по классификации Ижикевича [25].

Сравнение областей пачечной активности на плоскости $\left(g_{\mathrm{Na}}, I\right)$ до и после модификации натриевых $\mathrm{Na}_{V} 1.8$ каналов дано на рис. 5. До модификации этих каналов существуют две области пачечной активности (рис. 5,a). Левая область (ограниченная штриховыми линиями) занимает приблизительно $1 / 4$ часть левой области ритмической активности. Правая область пачечной активности составляет примерно 1/5 часть правой области ритмической активности. Следовательно, пачечная активность наблюдается при значениях стимулирующего тока от $I=16 \mu \mathrm{A} / \mathrm{cm}^{2}$ до $I=27 \mu \mathrm{A} / \mathrm{cm}^{2}$ и от $I=33 \mu \mathrm{A} / \mathrm{cm}^{2}$ до $I=57 \mu \mathrm{A} / \mathrm{cm}^{2}$.

После модификации параметров активационной воротной системы медленных тетродотоксин-устойчивых натриевых каналов $\mathrm{Na}_{V} 1.8$, вызванной действием 5-гидрокси- $\gamma$-пирон-2-карбоновой (коменовой) кислоты, приводящей к уменьшению величины эффективного заряда до значения $Z_{\text {eff }}=4.7 \pm 0.2$, в физиологически значимых пределах параметров $g_{\mathrm{Na}}$ и $I$ остается только одна область ритмической активности на плоскости параметров $\left(I, g_{\mathrm{Na}}\right)$, так как для второй области значения $g_{\mathrm{Na}}$ становятся отрицательными (рис. $5, b$ ). Таким образом, правая область для модифицированной системы практически исчезает. Соответственно уменьшается диапазон значений стимула, в котором возможны повторные ответы. Другими словами, после модификации $\mathrm{Na}_{V} 1.8$-каналов коменовой кислотой импульсная активность подавляется при больших значениях стимулирующего тока.

\section{Выводы}

Бифуркационный анализ модели мембраны нейрона дорсального ганглия позволил выявить возможность анальгетического подавления эктопических пачечных разрядов в ноцицептивных нейронах и получить ответ на вопрос о том, какие изменения в параметрах медленных натриевых каналов $\mathrm{Na}_{V} 1.8$ оказывают наибольшее влияние на передачу ноцицептивных сигналов. 


\section{Список литературы}

[1] Boucher T.J., McMahon S.B. // Curr Opin Pharmacol. 2001. P. 66-72.

[2] Michaelis M., Liu X.-G., Janig W. // J. Neurosci. 2000. Vol. 20. P. 2742-2748.

[3] Amir R., Michaelis M., Devor M. // J. Neurosci. 2002. Vol. 22. P. $1187-1198$

[4] Izhikevich E.M., Desai N.S., Walcott E.C., Hoppensteadt F.C. // Trends in Neuroscience. 2003. Vol. 26. P. 161-167.

[5] Devor M. // Exp. Brain. Res. 2009. Vol. 196. P. 115-128.

[6] Cummins T.R., Dib-Hajj S.D., Waxman S.G. // J. Neurosci. 2004. Vol. 24. P. 8232-8236.

[7] Herzog R.I., Cummins T.R., Ghassemi F. et al. // J. Physiol. 2003. Vol. 551. P. 741-750.

[8] Lossin C., Rhodes T.H., Desai R.R. et al. // J. Neurosci. 2003. Vol. 23. P. 11289-11295.

[9] Mantegazza M., Yu F.H., Powell A.J., Clare J.J. et al. // J. Neurosci. 2005. Vol. 25. P. 3341-3349.

[10] Renganathan M., Cummins T.R., Hormuzdiar W.N. et al. // J. Neurophysiol. 2000. Vol. 84. P. 710-718.

[11] Kerr N.C., Gao Z., Holmes F.E. et al. // Mol. Cell. Neurosci. 2007. Vol. 35. P. 283-291.

[12] Liu C.N., Michaelis M., Amir R. et al. // J. Neurophysiol. 2000. Vol. 84. P. 205-215.

[13] Bevan S., Storey N. // Novartis. Found. Symp. 2002. Vol. 241. P. 144-153.

[14] Wu N., Enomoto A., Tanaka S. et al. // J. Neurophysiol. 2005. Vol. 93. P. 2710-2722.

[15] Lai J., Porreca F., Hunter J.C. et al. // Ann. Rev. Pharmacol Toxicol. 2004. Vol. 44. P. 371-397.

[16] Waxman S.G., Cummins T.R., Dib-Hajj S.D. et al. // J. Rehabil Res. Dev. 2000. Vol. 37. P. 517-528.

[17] Ogata N., Ohishi Y. // Jpn. J. Pharmacol. 2002. Vol. 88. P. 365377.

[18] Kovalsky Y., Amir R., Devor M. // J. Neurophysiol. 2009. Vol. 102. P. $1430-1442$.

[19] Дик О.Е., Шелых Т.Н., Плахова В.Б., Ноздрачев А.Д. и др. // ЖТФ. 2015. Т. 85. С. 131-134.

[20] Plakhova V.B., Rogachevsky I.V., Lopatine E.V. et al. // Act. Nerv. Super Rediviva. 2014. Vol. 56. P. 55-64.

[21] Kuznetsov Y.A. Elements of Applied Bifurcation Theory. NY: Springer-Verlag, 1995.

[22] Dhooge A., Govaerts W., Kuznetsov Y.A. et al. MatCont and CL_Matcont Continuation toolboxes in MATLAB, Netherlands: Utrecht Univ, 2006.

[23] Guckenheimer J., Harris-Warrick R.M., Peck J. et al. // J. Comp. Neurosci. 1997. Vol. 4. P. 257-277.

[24] Guckenheimer J., Holmes D. Nonlinear Oscillations, Dynamical Systems, and Bifurcations of Vector Fields, NY: Springer-Verlag, 1983.

[25] Izhikevich E.M. // J. Bifur. and Chaos. 2000. Vol. 10. P. 11711266.

[26] Kovalsky Y., Amir R., Devor M. // Exp. Neurol. 2008. Vol. 210. P. 194-206. 